\title{
Education and regulation
}

\begin{abstract}
Fibre-optic sensors have been around for many years, but their market penetration has been slow. Nadya Anscombe talks to Brian Culshaw of Strathclyde University in the UK to find out why.
\end{abstract}

\begin{abstract}
What are the current trends in the optical-fibre-sensor market? The optical-fibre-sensor market is amazingly diverse, so identifying trends is difficult. However, one notable point is that market penetration has been, from a researcher's perspective, a bit disappointing. Fibre sensors have the potential to bring significant operational advantages to many applications, but the sensors are often used in industries that are very conservative. Many of the underlying technologies and principles have not changed much since optical-fibre sensors were invented gratings are still gratings, and fibres are still fibres - we have, however, seen many improvements in applications engineering. Systems for the interrogation of fibre Bragg gratings have improved, for example, and the use of spectroscopic signatures has become more sophisticated. In fact, much of the technological development has been at the detector and signal-processing end, rather than within the sensors themselves.
\end{abstract}

\section{What needs to happen to speed up market penetration?}

Education and regulation. These aspects have nothing to do with the science and technology of sensors, so often get overlooked by engineers, but education and standards are very important. The people who could use the technology need to be educated in how to use it and what benefits it can bring; and we need to establish standards and regulations to ensure a common language and understanding. Getting industry to take on a new technology can be an arduous task. Modern aircraft and many satellites now use optical-fibre gyroscopes for navigation and stabilization, but the evolution from mechanical spinning-mass systems took 20 years. Fibre gyroscopes succeeded because they are less dependent on very-high-precision mechanics and offer a much wider range of operating conditions. The key to success with optical-fibre sensors is finding those applications where the fibre sensor can do things that other sensors cannot.

Regulations are needed because nobody measures something unless they have to or they perceive a significant

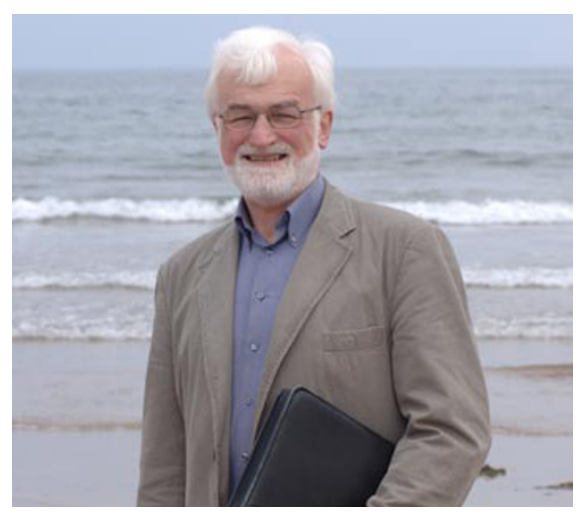

Sensor expert: Brian Culshaw.

economic advantage. For example, there have been several instances of bridges collapsing. These could possibly have been avoided if the structural health of those bridges had been continuously monitored. However, there is no regulation in place that states the structural health of a building needs to be continuously monitored so construction companies and bridge owners avoid the expense.

Two other important aspects of fibre-sensor development, which many technologists under-rate, are packaging and interface. It is important to collaborate with the people who will be using their products to find out how the product should be packaged and how the interface can be designed to make it user-friendly. If the developers get this right, customers will be more likely to buy the technology.

\section{What are the most interesting} emerging applications?

The aerospace industry is starting to use or investigate fibre-optic sensors. But it is a very conservative industry and, although I find this frustrating, I am pleased by this conservatism every time I fly. However, I do believe fibre-optic technology will succeed in the aerospace industry. The timing will depend on how the technology will fit into the regulatory framework and on the perceived benefit to manufacturers and passengers.
The technology is making inroads into medical instruments, and can be found at the heart of optical-coherence-tomography probes, producing unparalleled images from within the eye and immediately below the skin. Industrial applications include the monitoring of currents and voltages in overhead electrical power transmission lines; looking at concentrations of hazardous gases in the atmosphere; monitoring gas concentrations in hydrogen fuel cells; and discerning food quality through highly precise colour measurements. In all of these applications, however, fibre-optic sensors are used in relatively modest total volumes.

One of the great advantages that fibre-optic sensors have over other types of sensors is that they can be networked and perform distributed measurements. For example, they can be used in tunnels over many tens of kilometres mapping temperature changes with a resolution of about one metre, making them ideal for monitoring outbreaks of fire. A recent report by the Optoelectronics Industry Development Association (OIDA) in the USA is very optimistic that the prospects offered by distributed techniques will soon totally dominate fibre-sensor applications.

\section{What will be the most significant} developments over the next few years? The majority of present fibre sensors are based on very well established concepts, such as interferometry, diffraction and spectrometry. At this level, little is likely to change. However as an optical community we are continuing to perfect ever more elegant tools to handle light. It certainly isn't clear at present where new concepts, such as photonic crystals, slow light and all-optical switches, may find applications in sensing. However, as we learn more about how to manipulate light, these techniques will find their roles in sensor technology. We need to be well versed in the progress in optical techniques and technologies outside our own specialist fields and be able to spot the opportunity to use them.

Nadya Anscombe is a freelance science and technology journalist based in the UK. 